\title{
Développement d'un modèle de durabilité de poutres composites unidirectionnelles renforcées par des fibres de verre
}

\author{
V. Pauchard' ${ }^{1}$, H. Boulharts-Campion' ', F. Grosjean' ', P. Odru' et A. Chateauminois $2^{*}$ \\ 1 Institut français du pétrole, 1 et 4 avenue de Bois-Préau, 92852 Rueil-Malmaison Cedex - France \\ 2 École centrale de Lyon, Laboratoire IfoS, BP 163, 69131 Écully Cedex - France \\ e-mail : vincent.pauchard@ifp.fr - habiba.campion@ifp.fr - françois.grosjean@ifp.fr - pierre.odru@ifp.fr - antoine.chateauminois@espci.fr \\ *Pour toute correspondance relative à cet article : École supérieure de physique et chimie industrielles (ESPCI), Laboratoire PCSM \\ 10, rue Vauquelin, 75231 Paris Cedex 05 - France
}

\begin{abstract}
Résumé — Les interactions entre le vieillissement humide de composites unidirectionnels verre/époxy et leur tenue en fatigue par flexion sont ici abordées dans le cadre d'un modèle de durabilité fondé sur des notions de corrosion sous contrainte. L'étude est focalisée sur les stades initiaux de l'endommagement par fatigue, qui sont caractérisés par l'accumulation diffuse, à l'échelle microscopique, des ruptures différées des monofilaments de verre constituant le renfort. Ces processus sont décrits à l'aide d'un modèle de corrosion sous contrainte faisant appel, d'une part, à la distribution initiale des défauts de surface des fibres, d'autre part, à la cinétique de croissance sous-critique de ces mêmes défauts sous l'action combinée des contraintes et de l'humidité. Ces aspects sont validés expérimentalement sur des mèches de fibres non imprégnées, puis à l'échelle d'un volume élémentaire représentatif de composite. L'existence d'une relation empirique entre la densité de fibres rompues et la raideur en flexion trois points du matériau permet alors d'établir des expressions prédictives de la raideur. Celles-ci sont déduites du calcul de la probabilité de survie des fibres de verre. Le modèle aboutit à la détermination des cinétiques de perte de raideur de poutres de flexion composites en fonction de la nature du chargement mécanique de fatigue (niveau de déformation, fréquence, rapport de sollicitation) et des paramètres environnementaux (température, humidité). Les prédictions sont confrontées à des résultats expérimentaux obtenus sous chargements monotones, quasi statiques (relaxation) et de fatigue cyclique.
\end{abstract}

Mots-clés : durabilité, poutres composites, fibres de verre, fatigue, flexion, humidité, vieillissement, corrosion sous contrainte.

\begin{abstract}
Development of a Durability Model Applied to Unidirectional Composites Beams Reinforced with Glass Fibers - The interactions between hygrothermal ageing and the flexural fatigue behavior of unidirectional glass/epoxy composites are investigated within the frame of a Stress Corrosion Cracking model. The study is focused on the initial damage development stages, i.e. when the degradation mainly consists in the non interactive accumulation of delayed fibers failures at the microscopic scale. These processes are described on the basis of a knowledge of the initial fibers surface defects distribution and from the determination of the subcritical crack growth rates of these surface flaws under the combined action of moisture and stress. The stress corrosion behavior of the glass reinforcement is established experimentally using successively unimpregnated fibers bundles and microscopic observations of a composite elementary volume under tensile stresses. An empirical relationship between the broken fibers density and the flexural stiffness allows to predict the macroscopic stiffness loss from the
\end{abstract}


calculation of the fibers survival probability. The model accounts for the activation of the stiffness loss kinetics as a function of the fatigue (strain level, frequency, strain ratio) and environmental (temperature, humidity) parameters. Theoretical predictions are compared to experiments carried out under monotonic, relaxation and cyclic fatigue loading.

Keywords: durability, composites beams, glass fibers, bending, humidity, ageing, Stress Corrosion Cracking.

\section{INTRODUCTION}

Pour beaucoup d'applications structurales, comme sur les plates-formes pétrolières offshore, les composites à matrice polymère apparaissent comme de sérieux concurrents des métaux, du fait principalement de leur légèreté et de leur insensibilité à la corrosion saline. Pourtant, l'industrialisation de solutions composites est délicate, car les avantages compétitifs sont amoindris par les surcoûts induits, et surtout, par la difficulté à prévoir l'évolution des propriétés d'usage de ce type de matériaux, notamment sous sollicitations environnementales et mécaniques couplées.

Ces difficultés proviennent essentiellement du caractère hétérogène du matériau qui conduit, au cours de sa durée de vie, à l'interaction entre de nombreux mécanismes physicochimiques et mécaniques se produisant sur des échelles spatiales et temporelles très étendues [1]. Ceux-ci peuvent impliquer aussi bien le renfort, notamment quand il s'agit de fibres de verre réputées pour leur sensibilité à l'humidité, que la matrice (plastification, hydrolyse) ou l'interface (décohésion). Dans un tel contexte, il est extrêmement délicat, voire dangereux, d'identifier des lois de comportement phénoménologiques à partir d'essais dits « accélérés », consistant à accroître artificiellement les paramètres d'activation de la dégradation (contrainte, température, hygrométrie, etc.) afin d'obtenir des résultats dans un temps compatible avec le développement de la structure. Cette « accélération » peut effectivement induire une activation différentielle des mécanismes compétitifs ou coopératifs et, ainsi, conduire à une dénaturation de la cinétique macroscopique de dégradation des propriétés.

Dans certains cas, il est cependant possible d'identifier un mécanisme d'endommagement majoritaire (ou critique) à partir duquel un modèle physique de durabilité peut être élaboré en explicitant la cinétique de dégradation microscopique. Le cas pratique qui nous intéresse est a priori favorable à une telle approche. Il s'agit d'une structure autoportée « fibres de verre/matrice époxy », conçue à l'IFP, pour être incluse dans des canalisations conduisant le brut du fond marin à la plate-forme d'exploitation. Les sollicitations de traction uniaxiale y sont variables mais dominantes et le matériau retenu est un unidirectionnel sollicité parallèlement aux fibres (UD $0^{\circ}$ ). Ces dernières assurant l'essentiel des propriétés de rigidité et de résistance, leur rupture contrôle $a$ priori la déchéance de la structure [2]. Il est par ailleurs largement établi que dans des conditions de vieillissement humide, l'altération des propriétés du renfort en verre peut conduire à une réduction très importante des durées de vie sous sollicitation en fatigue [1, 3-6]. Les explications avancées font appel, soit à la création hydrolytique de défauts à la surface du verre $[7,8]$, soit à l'activation des processus de corrosion sous contrainte des fibres de renfort $[5,6,9,10]$. Dans ce dernier cas, l'accélération des vitesses de croissance souscritique des défauts conduit à une augmentation importante des ruptures différées des fibres sous chargement mécanique.

Les études existant à ce sujet indiquent cependant que l'abattement des propriétés mécaniques ne peut être simplement déduit de la quantité d'eau ayant diffusé dans le composite, mais qu'il faut prendre en compte l'existence d'un couplage entre le temps, la température et le niveau de sollicitation [11]. L'objectif de cette étude était d'expliciter ce couplage dans les hypothèses d'un modèle de corrosion sous contrainte (CSC), afin de pouvoir in fine rendre compte des cinétiques de perte de raideur du composite soumis à des sollicitations de fatigue en environnement humide. Ce travail a été mené pour des sollicitations de flexion trois points induisant un endommagement par traction sur la face tendue des éprouvettes composites unidirectionnelles. Outre sa simplicité de mise en œuvre, notamment dans le cas d'essais de fatigue, ce type de chargement présente l'avantage de conduire à une localisation de l'endommagement, mise à profit lors de l'analyse microscopique des mécanismes de rupture des fibres.

Le développement du modèle s'est appuyé sur une approche multiéchelle très progressive intégrant les étapes suivantes :

- Identification des mécanismes de corrosion sous contrainte et de leurs paramètres cinétiques à l'échelle des monofilaments de verre. De façon graduelle, ces aspects ont été abordés sur fibres de verre non imprégnées, puis au sein d'un volume élémentaire représentatif (VER) du composite. Cette première étape a abouti à la définition d'un modèle de CSC permettant de décrire la cinétique microscopique d'accumulation des ruptures de fibres à l'échelle du VER.

- Détermination d'une relation micro/macro permettant de relier l'endommagement microscopique du VER à la perte de raideur macroscopique de la poutre composite sollicitée en flexion trois points. La modélisation complète d'une telle relation sortant des objectifs de ce travail, cette étape 
a été abordée de façon empirique en corrélant les mesures de perte de raideur aux densités de fibres rompues détectées dans le VER.

- Transposition à l'échelle de la poutre du modèle de CSC et application à des cas de chargement de complexités croissantes : sollicitations monotones, relaxation, fatigue cyclique et fatigue à spectre de chargement. L'objectif était ici de définir l'aptitude du modèle à prédire l'activation des cinétiques de pertes de raideur en fonction du niveau et de la fréquence de sollicitation ainsi que des paramètres environnementaux (humidité, température).

L'ensemble de cette approche a été appliqué à la prévision des stades initiaux de l'endommagement par fatigue, c'est-àdire avant l'apparition d'un endommagement macroscopique significatif en termes de fissuration ou de délaminage. Limitées à des pertes de raideur de 5 à $10 \%$, ces étapes de premier endommagement sont dominées par les ruptures différées et diffuses des fibres de verre à l'échelle microscopique. Moyennant cette restriction sur la nature des mécanismes de dégradation, il est dans une large mesure possible d'assimiler le comportement longitudinal du composite unidirectionnel à celui d'un écheveau de monofilaments indépendants soumis à des sollicitations de traction. Cette hypothèse constitue le cœur du modèle proposé et permet de ramener le calcul de la raideur résiduelle à celui de la probabilité de survie d'une population statistiquement représentative de fibres au sein du composite. Ce calcul requiert principalement la connaissance de la distribution initiale des défauts à la surface de la fibre, ainsi que la loi de propagation sous-critique de ces défauts dans l'environnement considéré.

\section{BASES THÉORIQUES DU MODĖLE DE CORROSION SOUS CONTRAINTE}

Dans le cas du verre massif, il est largement établi que la vitesse de croissance sous-critique, $v$, d'un défaut en mode $I$ peut être reliée au facteur d'intensité de contrainte, $K_{I}$, par une relation du type :

$$
v=\frac{d a}{d t}=A K_{I}^{n}
$$

avec

$$
K_{I}=Y \sigma \sqrt{a}
$$

où $a$ est la longueur de fissure, $\sigma$ est la contrainte appliquée et $Y$ un facteur de forme voisin de $\sqrt{\pi}$. Les deux paramètres, $A$ et $n$, de la loi de propagation sous-critique sont généralement considérés comme constants dans un environnement physicochimique donné.

Il convient de noter que le domaine de validité de l'équation (1) est restreint à des valeurs du facteur d'intensité de contrainte correspondant au stade I, c'est-à-dire comprises entre le seuil de non-propagation, $K_{I S}$, et le stade II où le facteur limitant la cinétique de fissuration devient la diffusion des espèces réactives en fond de fissure. En stade I, la vitesse d'avancée de la fissure est au contraire supposée contrôlée par la cinétique de rupture intrinsèque des liaisons interatomiques du réseau silicate en présence d'eau.

Les stades de propagation II et III (rupture fragile) correspondent à des temps de rupture très courts ne présentant que peu d'intérêt du point de vue de la durée de vie en fatigue d'une pièce. Leur contribution peut donc être négligée dans l'estimation des temps à rupture. Par intégration de l'équation (1), il est alors possible d'exprimer le temps à rupture, $t_{f}$, d'une pièce sous une contrainte appliquée, $\sigma(t)$ :

$$
\int_{0}^{t_{f}} \sigma(t)^{n} d t=\frac{2 K_{I C}^{2-n}}{A Y^{2}(n-2)} \sigma_{i}^{n-2}
$$

avec $K_{I C}$, la ténacité du verre, $\sigma_{i}$, la résistance mécanique de la pièce considérée [12], telle qu'elle aurait pu être mesurée en atmosphère inerte, c'est-à-dire en l'absence de mécanismes de corrosion sous contrainte. Dans le cas de sollicitations périodiques, le premier terme de l'équation (3) correspond en fait à l'intégrale d'une fonction périodique sur un intervalle borné. Pour des valeurs suffisamment élevées de $t_{f}$ $\begin{array}{lll}i & e & 0\end{array}$, $t_{f} \gg 1 / v$ où $v$ est la fréquence de sollicitation), on peut alors facilement démontrer que l'expression (3) se simplifie de la façon suivante :

$$
t_{f}=\frac{2 K_{I C}^{2-n} \sigma_{i}^{n-2}}{A Y^{2}(n-2) \lambda \sigma_{\max }^{n}}
$$

avec

$$
\lambda=\frac{1}{t} \int_{0}^{t}\left(\frac{\sigma(t)}{\sigma_{\max }}\right)^{n} d t
$$

où $\sigma_{\max }$ est la valeur maximale de la contrainte appliquée. $\lambda$ est donc une constante numérique inférieure à 1 et dépendant de la sollicitation appliquée et de $n$. Sous sollicitation statique (pour le verre, en fluage ou relaxation, $\sigma=E \varepsilon=$ cste, donc le rapport de sollicitation $r=\sigma_{\min } / \sigma_{\max }$ vaut 1$), \lambda=1$. Ce calcul démontre donc que, si le modèle de corrosion sous contrainte est approprié, le temps à rupture d'une pièce doit être une fonction croissante de sa résistance initiale. Dans le cas d'une population d'éprouvettes dont les défauts de surface sont distribués statistiquement, il est donc possible d'associer la distribution des contraintes à ruptures en milieu inerte à la distribution des durées de vie.

De nombreux travaux $[13,14]$ ont démontré que la statistique de Weibull [15] constituait un cadre approprié pour décrire la distribution des valeurs de $\sigma_{i}$ de matériaux fragiles 
tels que le verre. Selon cette approche, la probabilité de survie, $P_{s}$, à la contrainte $\sigma_{i}$ s'écrit :

$$
P_{s}\left(\sigma_{i}\right)=\exp \left[-\left(\sigma_{i} / \sigma_{0}\right)^{m}\right]
$$

où $m$ est le module de Weibull caractérisant la largeur de la distribution et $\sigma_{0}$ un facteur d'échelle dépendant du volume de matière sollicité. En substituant (4) dans (6), il vient donc :

$$
P_{s}(t)=\exp \left[-t^{m / n-2} \lambda^{m / n-2} \sigma_{\max }^{m n / n-2} C\right]
$$

avec

$$
C=\left[\frac{(n-2) A Y^{2}}{2 K_{I C}^{2-n} \sigma_{0}^{n-2}}\right]^{m / n-2}
$$

Dans le cadre de l'étude, cette approche sera appliquée à des ensembles statistiquement représentatifs de fibres de verre supposées parallèles et soumises à des sollicitations de traction à déformation imposée. Après réécriture en déformation de l'équation (7) il vient :

$$
P_{s}(t)=\exp \left[-t^{m / n-2} \lambda^{m / n-2} \varepsilon_{\max }^{m n / n-2} k\right]
$$

avec

$$
k=\left(\frac{E^{n}(n-2) A Y^{2}}{2 K_{I C}^{2-n} \varepsilon_{0}^{n}}\right)^{m / n-2}
$$

où $E$ est le module de Young de la fibre et $\varepsilon_{0}$ le paramètre d'échelle de la loi de Weibull.

Sous réserve de détermination de la constante $k$, l'expression ci-dessus permet donc théoriquement de déterminer l'activation des cinétiques de rupture de fibres en milieu humide en fonction du chargement périodique ou quasi statique appliqué. En plus des caractéristiques mécaniques du matériau (module et ténacité), le modèle nécessite l'identification des paramètres $n$ et $m$ caractérisant respectivement la cinétique de propagation sous-critique des défauts et la distribution initiale des défauts à la surface des fibres. Le module de Weibull $m$ peut classiquement être déterminé à partir d'essais de traction sur monofilaments ou mèches de fibres [16]. La valeur de $n$ peut, quant à elle, être déduite de la pente du tracé $\log \left(-\ln \left(P_{s}\right)\right)=f(\log (t))$ qui doit être linéaire si l'équation (8) est vérifiée. Cette dernière méthode reste cependant imprécise [17] et on lui préférera dans cette étude l'identification de $n$ à partir de la courbe donnant la vitesse de propagation sous-critique en fonction de $K_{I}$.

Ce modèle d'écheveau de fibres soumises à des déformations uniformes de traction constitue la base de notre modèle de CSC. Il sera successivement validé à l'échelle d'une mèche de fibres non imprégnée, puis d'un volume élémentaire de composite, avant d'être transposé au suivi de raideur de la poutre de flexion en composite.

\section{MISE EN ÉVIDENCE DE LA CORROSION SOUS CONTRAINTE DES FIBRES DE VERRE}

\subsection{Propagation sous-critique de défauts dans des fibres de verre non imprégnées}

Une étape préliminaire dans le développement du modèle de durabilité a consisté à identifier précisément la nature des mécanismes de rupture de monofilaments de verre en milieu humide, à partir d'une détermination expérimentale de la courbe $v=f\left(K_{I}\right)$. En première intention, le plus simple pour tracer une courbe de propagation est de mesurer, au cours du temps, la longueur d'une fissure sous chargement constant [18]. À l'échelle de monofilaments d'une dizaine de micromètres de diamètre, la mise en œuvre de cette approche est cependant particulièrement délicate et restreinte à des défauts pouvant être observés optiquement.

Une solution alternative est de considérer que la durée de vie d'une éprouvette sous sollicitation quasi statique contient la loi de propagation sous une forme intégrale [19]. Cette approche permet d'expliciter la relation $v\left(K_{I}\right)$ à partir de données plus facilement accessibles à l'expérimentation, à savoir des distributions de résistances initiales et de temps à rupture sous chargement statique. D'après (2), il vient en effet :

$$
t_{f}=\int_{0}^{t f} d t=\int_{a_{0}}^{a_{f}} \frac{d a}{v}=\frac{2}{\sigma_{\mathrm{app}}^{2} Y^{2}} \int_{K_{I 0}}^{K_{I F}} \frac{K_{I} d K_{I}}{v\left(K_{I}\right)}
$$

avec $K_{I 0}=\sigma_{\text {app }}\left(a_{0}\right)^{1 / 2} Y$ le facteur d'intensité de contrainte initial, et $\sigma_{\text {app }}$ la contrainte appliquée. $K_{I F}$ et $a_{f}$ sont respectivement la valeur du facteur d'intensité de contrainte et la taille du défaut au moment de la rupture. Sans hypothèse sur $v\left(K_{I}\right)$ on peut dériver cette intégrale par rapport à $K_{I 0}$ :

$$
\frac{d t_{f}}{d K_{I 0}}=-\frac{2 K_{I 0}}{\sigma_{\mathrm{app}}^{2} Y^{2} v\left(K_{I 0}\right)}
$$

or

donc

$$
K_{I 0} / K_{I C}=\sigma_{\text {app }} / \sigma_{i}
$$

$$
d K_{I 0}=\frac{-K_{I C} \sigma_{\mathrm{app}}}{\sigma_{i}^{2}} d \sigma_{i}
$$

ce qui donne :

$$
v\left(K_{I 0}\right)=\frac{2 K_{I C}^{2}}{\varepsilon_{i}^{3} E^{2} Y^{2}} \frac{d \varepsilon_{i}}{d t_{f}}
$$

Pour tracer la courbe $v=f\left(K_{I}\right)$, il suffit donc de pouvoir déterminer les résistances initiales, $\varepsilon_{i}$, d'une série d'échantillons en fonction de leur temps à rupture, $t_{f}$. À défaut de pouvoir réaliser un essai monotone et un essai statique sur la 
même fibre, on peut s'appuyer sur des considérations statistiques. Si deux lots distincts de fibres sont de tailles suffisantes pour être statistiquement représentatifs, ils seront identiques en termes de distribution des défauts de surface. La distribution des résistances mécaniques obtenue sur un lot correspondra donc, terme à terme, à la distribution des temps à rupture obtenue sur l'autre. Une série de couples $\left(\varepsilon_{i}, t_{f}\right)$ peut ainsi être générée en appariant les deux distributions.

Lors d'essais de traction réalisés sur mèches, des techniques d'émission acoustique détaillées par ailleurs [20] nous ont permis de détecter individuellement les ruptures de fibres au sein des mèches. Plus de 2000 monofilaments sont ainsi caractérisés simultanément lors d'un seul essai et l'hypothèse de représentativité statistique est donc largement remplie. Sur la base d'essais monotones réalisés en atmosphère inerte et d'essais quasi statiques à déformation imposée réalisés dans différents environnements, nous avons ainsi pu établir de façon originale des courbes $v\left(K_{I}\right)$ sur des monofilaments de verre $\mathrm{E}_{\mathrm{CR}}{ }^{\circledR}$ (Owens Corning) [21, 22]. La figure 1 en donne un exemple à température ordinaire pour 10 et $50 \%$ d'humidité relative (HR).

Les deux tracés présentent toutes les caractéristiques associées aux mécanismes de CSC, à savoir, des stades I parallèles au-dessus de seuils très apparents, dont la valeur diminue avec l'augmentation du degré d'hygrométrie. Aux forts facteurs d'intensité de contrainte, les courbes se rejoignent, ce qui permet de positionner le stade III. Entre les deux, le stade II est plus difficile à situer visuellement : très court à $50 \% \mathrm{HR}$, il est quasi inexistant à $10 \% \mathrm{HR}$. On peut noter aussi qu'à $50 \% \mathrm{HR}$, la vitesse palier est d'environ $10^{-8} \mathrm{~m} / \mathrm{s}$, c'est-à-dire inférieure de trois à quatre ordres de grandeur aux valeurs courantes pour le verre [13]. Ces deux observations peuvent être significatives du fait que le verre n'est

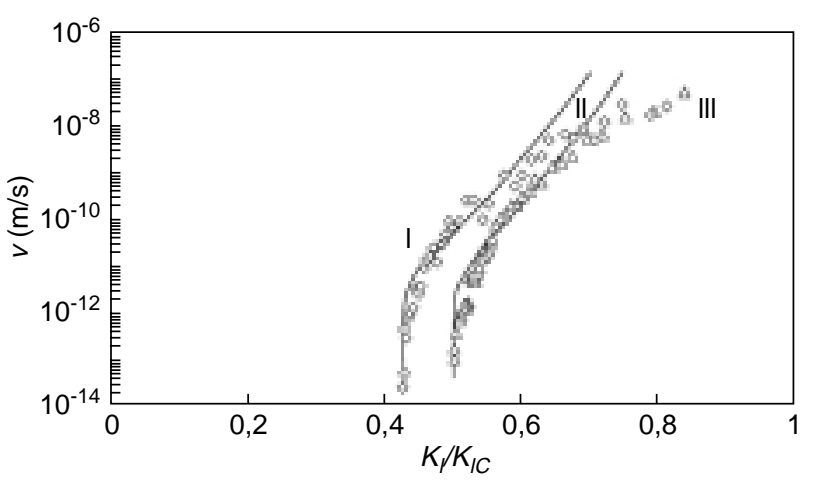

Figure 1

Courbe $v\left(K_{I}\right)$ sur mèches de verre $E_{C R}$ à $10 \%(\Delta)$ et $50 \%(\diamond)$ d'humidité relative.

$v\left(K_{I}\right)$ plot for $E_{C R}$ glass fibers under $10 \%(\Delta)$ and $50 \%(\diamond)$ relative humidity. réellement exposé qu'aux quantités d'eau diffusant à travers le produit d'ensimage. La valeur du paramètre de corrosion sous contrainte, $n$, estimée à partir du stade I est voisine de 20 , soit un résultat proche de celui obtenu par Cowking et. al [10] sur du verre $E$ en immersion dans l'eau. $n$ est par ailleurs indépendant du degré d'hygrométrie conformément aux références bibliographiques sur le verre massif [23]. Ces expériences permettent donc sans ambiguïté d'établir que la corrosion sous contrainte est bien le mécanisme physique responsable de la rupture différée des fibres en milieu humide.

\subsection{Ruptures différées de fibres dans un volume élémentaire représentatif de composite}

Les mécanismes de corrosion sous contrainte étant sensibles à l'environnement physicochimique des fibres, les données obtenues sur mèches non imprégnées ne peuvent être directement transposées aux monofilaments renforçant la matrice polyépoxyde du composite. Des observations microscopiques in situ des processus de rupture des fibres ont donc été menées afin d'établir les paramètres cinétiques de la CSC au sein du composite vieilli.

Sous sollicitation de flexion, l'essentiel de l'endommagement est localisé sur la face en traction des éprouvettes, à l'aplomb de l'appui central, ce qui permet de restreindre les observations à une zone de quelques $\mathrm{mm}^{2}$. En jouant sur la transparence du composite, un dispositif spécifique d'acquisition d'images asservi en déplacement [3] nous a permis de quantifier le nombre de fibres rompues au sein de cette zone, en fonction du temps et du niveau de chargement (fig. 2). Ces analyses ont été menées sur des éprouvettes planes d'un composite modèle verre/époxy élaboré à l'IFP au moyen d'une installation pilote d'enroulement filamentaire. L'optimisation des conditions d'élaboration a conduit à l'obtention d'un matériau exempt de porosités (moins de $0,5 \%$ en volume) et de défauts d'imprégnation, permettant ainsi des conditions d'observations optimales.

Sur la base d'une étude préalable des mécanismes de diffusion de l'humidité [23], le conditionnement de ce composite a été défini de façon à assurer l'homogénéité de son état de vieillissement dans l'épaisseur observée, a priori délimitée par la profondeur de champ de l'objectif $(\sim 150 \mu \mathrm{m})$. Des mesures gravimétriques de sorption d'eau réalisées en immersion à $25^{\circ} \mathrm{C}$ ont permis d'évaluer le coefficient de diffusion du composite $\left(D=1,210^{-13} \mathrm{~cm}^{2} / \mathrm{s}\right)$ dans les hypothèses de la loi de Fick [24]. Dans ces conditions, un calcul indique que la saturation est atteinte dans les couches superficielles du composite au bout d'une dizaine de jours. À $25^{\circ} \mathrm{C}$, les courbes de sorption d'eau ne mettent par ailleurs pas en évidence de processus d'hydrolyse, ce qui permet de considérer les couches saturées comme homogènes sur le plan physicochimique. 


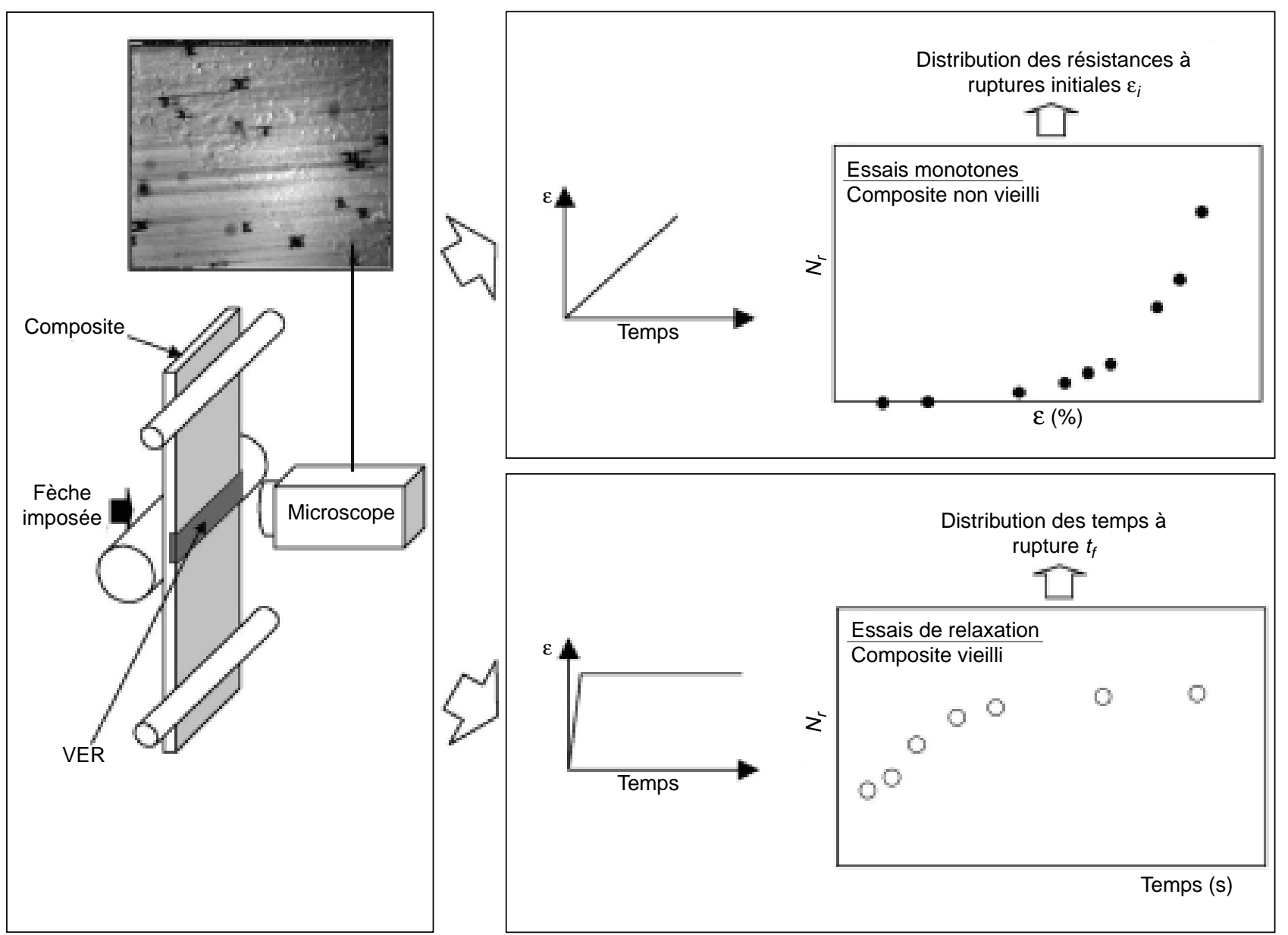

Figure 2

Quantification du nombre de fibres rompues, $N_{r}$, dans un volume élémentaire de poutre composite soumise à une sollicitation de flexion. Quantitative analysis of the number of broken fibers within a representative elementary volume of a flexural composite beam.

Une analyse micromécanique préalable [20] a démontré que le domaine observé constituait un volume élémentaire représentatif (VER) satisfaisant aux conditions suivantes :

- En dépit des gradients de contrainte inhérents à l'essai de flexion trois points, la déformation en traction des fibres au sein du VER peut être considérée comme homogène du point de vue de leur cinétique de rupture.

- Les effets de rechargement liés au transfert de charge à l'interface ne conduisent qu'à l'apparition d'un nombre très limité de fibres multifragmentées. En première approximation, on supposera donc que chaque fibre ne se rompt qu'une seule fois au sein du VER.

- Dans les conditions d'observations considérées, les effets d'interaction entre ruptures sont négligeables. Autrement dit, les concentrations de contraintes au voisinage des extrémités rompues des fibres n'induisent pas d'augmentation significative de la densité de monofilaments rompus.
L'ensemble de ces considérations conduit à assimiler le VER à un écheveau de fibres parallèles soumises à une déformation constante et à un environnement physicochimique homogène. Il devient alors possible d'appliquer aux données des observations microscopiques le formalisme décrit dans le premier chapitre et déjà appliqué aux mèches non imprégnées. Une inconnue supplémentaire réside cependant dans l'estimation de l'épaisseur de composite observée, c'est-à-dire du nombre total, $N_{t}$, de fibres du VER. Cette donnée est effectivement indispensable au calcul de la probabilité de survie $P_{s}=1-N_{r} / N_{t}$, où $N_{r}$ est le nombre de fibres rompues. Des considérations semi-quantitatives convergentes [20] nous ont permis de conclure que les observations optiques concernaient une à deux monocouches de filaments, soit un nombre de fibres compris entre 600 et 1200 . Les analyses décrites ci-après ont été effectuées selon cette hypothèse. 
À partir de caractérisations monotones sur composite non vieilli et d'essais de relaxation sur composite vieilli (fig. 2), nous avons pu déterminer les distributions de $\varepsilon_{i}$ et $t_{f}$ et donc établir les courbes $v\left(K_{I}\right)$ des monofilaments dans leur environnement physicochimique réel. La procédure utilisée est en tout point identique à celle mise en œuvre pour les essais de traction sur mèche et aboutit aux courbes représentées dans la figure 3. Quelle que soit l'hypothèse sur $N_{t}$, les courbes présentent un seuil de non-propagation et un stade I. L'absence de stade II et III est due au fait que l'acquisition d'image débute quelques minutes après la mise en charge. Comparativement aux mèches non imprégnées testées en humidité relative, l'influence de la matrice est principalement sensible sur la valeur du paramètre de corrosion sous contrainte, $n$, qui diminue de 23 à 11 . Le seuil de non-propagation, $K_{I S}$, est également sensiblement abaissé par rapport aux fibres non imprégnées à $50 \% \mathrm{HR}$ (fig. 1), ce qui démontre que la matrice ne joue pas de rôle d'agent protecteur des fibres visà-vis de l'environnement.

Les mécanismes de CSC étant clairement identifiés au sein du VER, les cinétiques expérimentales de rupture en milieu humide ont été confrontées au modèle détaillé dans la première partie. Ce dernier indique que la probabilité de rupture de la population de fibres dont le défaut létal se propage initialement en mode $I$ doit obéir à la relation (8). Par ailleurs, il est également possible de démontrer que $P_{s}$ doit tendre aux temps longs vers une limite asymptotique, $P_{\mathrm{s}}^{\infty}$ [23]. Pour une déformation donnée, la distribution des résistances mécaniques entraîne la distribution des facteurs d'intensité de contrainte :

$$
K_{I}=\frac{\varepsilon_{\mathrm{app}} K_{I S}}{\varepsilon_{i}}
$$

Au sein de l'écheveau, certaines des fibres se rompront (si $\varepsilon_{i}<K_{I S} \varepsilon_{\mathrm{app}} / K_{I}$ ), d'autres jamais (si $\varepsilon_{i}>K_{I S} \varepsilon_{\mathrm{app}} / K_{I}$ ). La valeur asymptotique de la probabilité de rupture est donc obtenue en injectant la déformation à rupture de la dernière fibre déchue dans l'expression décrivant la distribution des résistances mécaniques (éq. (6)) :

$$
P_{s}^{\infty}=\exp \left[-\left(\frac{\varepsilon_{\mathrm{app}} K_{I C}}{\varepsilon_{0} K_{I S}}\right)^{m}\right]
$$

L'application du modèle requiert la détermination des paramètres suivants :

- les valeurs des constantes $\varepsilon_{0}$ et $m$, caractérisant la distribution de Weibull des résistances initiales. Celles-ci sont déterminées d'après l'analyse expérimentale des résistances mécaniques des fibres au sein du VER non vieilli soumis à un chargement monotone (fig. 2);

- le paramètre de corrosion sous contrainte, $n$, identifié d'après le stade I de la courbe $v\left(K_{I}\right)$ du VER ;

- la constante $k$, ici considérée comme un facteur de calage, qui peut être identifiée d'après la distribution des temps à rupture d'un essai quasi statique de référence réalisé dans des conditions connues de déformation imposée et de vieillissement.

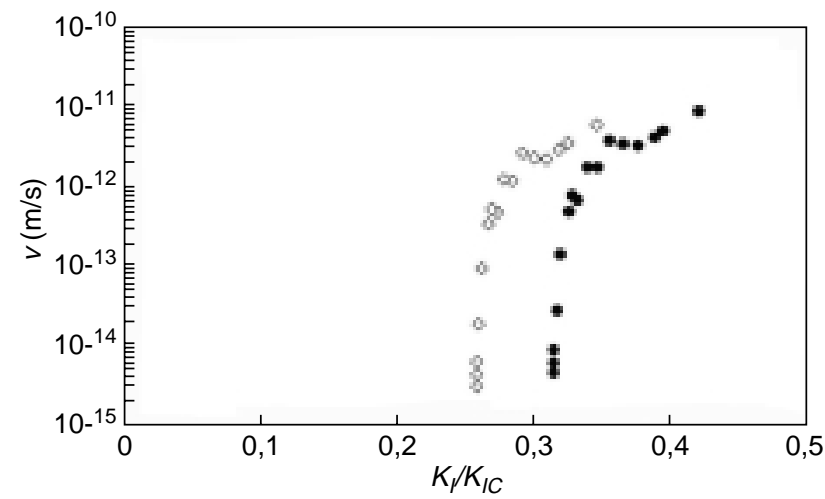

Figure 3

Courbes $v\left(K_{I}\right)$ des fibres de verre $\mathrm{E}$ Advantex ${ }^{\circledR}$ au sein d'un volume élémentaire représentatif (VER) de composite vieilli 10 jours à $25^{\circ} \mathrm{C}$.

Hypothèse sur le nombre $N_{t}$ de fibres contenues dans le VER: $(\diamond) N_{t}=600 ;(\bullet) N t=1200$.

$v\left(K_{I}\right)$ relationship for Advantex ${ }^{\circledR}$ E-glass fibers within an elementary composite volume aged 10 days at $25^{\circ} \mathrm{C}$.

Hypothesis for the number of fibers enclosed within the elementary composite volume: $(\diamond) N_{t}=600 ;(\bullet) N_{t}=1200$.

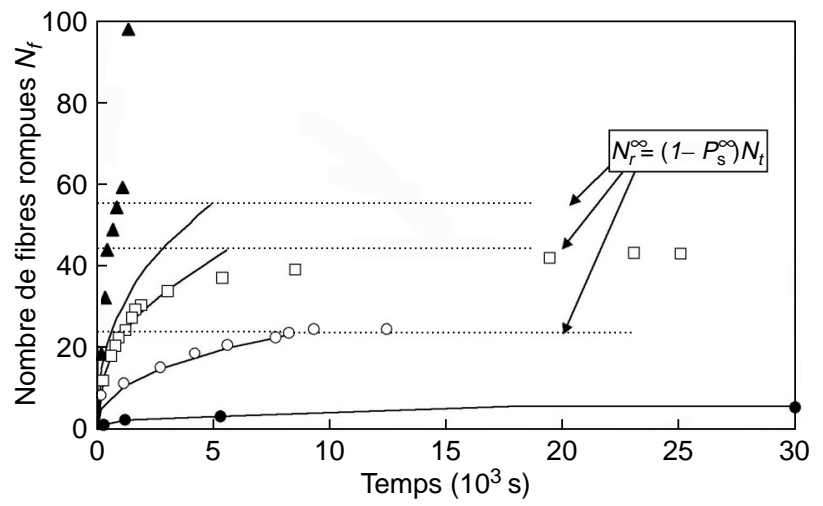

Figure 4

Cinétiques de rupture différée de fibres de verre $\mathrm{E}$ Advantex ${ }^{\circledR}$ au sein d'un volume élémentaire représentatif de composite vieilli soumis à une sollicitation de relaxation.

(Lignes continues : éq. (8)).

$(\bullet) \varepsilon_{\max }=1,0 \%$; (०) $\varepsilon_{\max }=1,5 \%$; (口) $\varepsilon_{\max }=1,8 \%$; (ム) $\varepsilon_{\max }=1,9 \%$.

Kinetics of delayed fibers failure of Advantex ${ }^{\circledR}$ E-glass fibres within an elementary composite volume under a relaxation condition.

(Solid lines: Eq. (8))

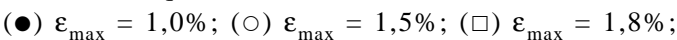
(A) $\varepsilon_{\max }=1,9 \%$. 
La figure 4 confronte la prévision à l'expérience pour quatre niveaux de déformation imposée, différents du niveau utilisé pour l'identification du paramètre de calage $k$. À $1,9 \%$ de déformation, la cinétique de rupture est plus rapide que prévue. Les observations optiques montrent que cet écart est associé à la formation rapide de zones critiques dans lesquelles les concentrations de contraintes en bout de fibres induisent des ruptures localisées en cascade qui sortent des hypothèses du modèle. Dans les autres cas, on observe un très bon accord avec la théorie, tant dans la première partie décrite par l'équation (8) qu'en ce qui concerne les temps longs décrits par la limite asymptotique $P_{s}^{\infty}$. L'hypothèse du quasi-écheveau de fibres et le formalisme afférent se trouvent donc confortés jusqu'à $1,8 \%$ de déformation et des temps de l'ordre de $2,510^{4} \mathrm{~s}$.

\section{RELATION MICRO/MACRO}

La densité de fibres rompues au sein d'un composite ne constitue pas une information exploitable dans une démarche de dimensionnement de structures. L'application du modèle de CSC implique donc de pouvoir relier cette information microscopique aux propriétés mécaniques résiduelles de la poutre de flexion. Cette démarche a été entreprise en considérant la raideur de la poutre comme un indicateur quantitatif du développement de l'endommagement microscopique.

Sur la base d'observations optiques sous sollicitations monotones et de relaxation, nous avons pu établir de façon empirique l'existence d'une relation linéaire entre la densité de fibres rompues dans le VER et la raideur résiduelle, $R / R_{0}$, de la poutre composite (fig. $5 a$ ). Le développement d'un modèle micromécanique détaillé permettant de justifier ce résultat sort du cadre de l'étude, mais une modélisation simplifiée par élément fini nous a toutefois permis de justifier le caractère linéaire de la relation $R / R_{0}=f\left(N_{r}\right)$ et les ordres de grandeur de la pente associée.
Le principe de la simulation repose sur la détermination de la raideur d'une poutre composite dans laquelle le module deYoung est distribué de façon à rendre compte de la dégradation du matériau, c'est-à-dire de la présence de fibres rompues (fig. $5 b$ ). En faisant coïncider la longueur des mailles du modèle «éléments finis » avec la longueur de rechargement aux extrémités des fibres (estimée à environ $150 \mathrm{~mm}$ ), on peut effectivement écrire en première approximation que le module endommagé est égal au produit $E_{0} P_{s}(\varepsilon)$, où $E_{0}$ est le module non endommagé du composite. Pour un niveau de flèche donné, le module associé à chaque maille du modèle de poutre est ainsi calculé à partir de la valeur locale de la déformation et de la statistique de Weibull

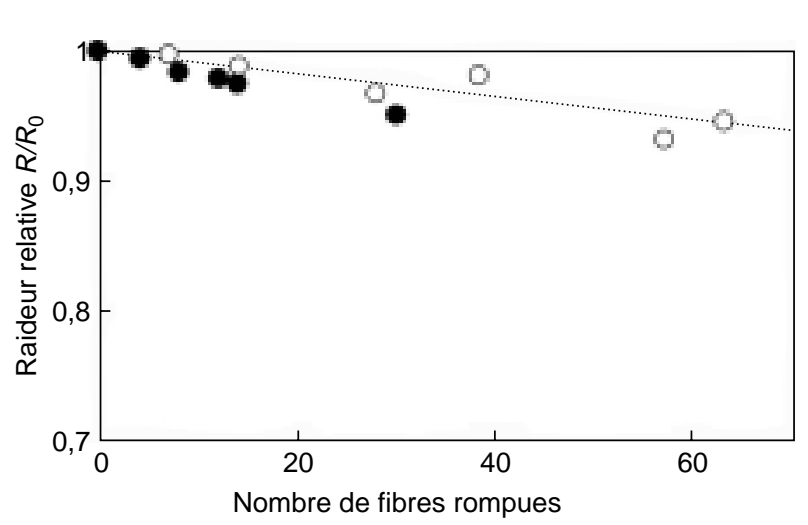

Figure 5a

Relation entre raideur macroscopique et nombre de fibres rompues dans un VER de poutre unidirectionnelle soumise à une sollicitation de flexion trois points.

(•) essais de relaxation ; (O) essais monotones ; ligne pointillée : simulation numérique.

Relationship between the macroscopic stiffness and the number of broken fibers within an elementary volume of a UD composite beam under a three-point bending condition.

(•) relaxation tests; (O) monotonic tests; dashed line: numerical simulation.

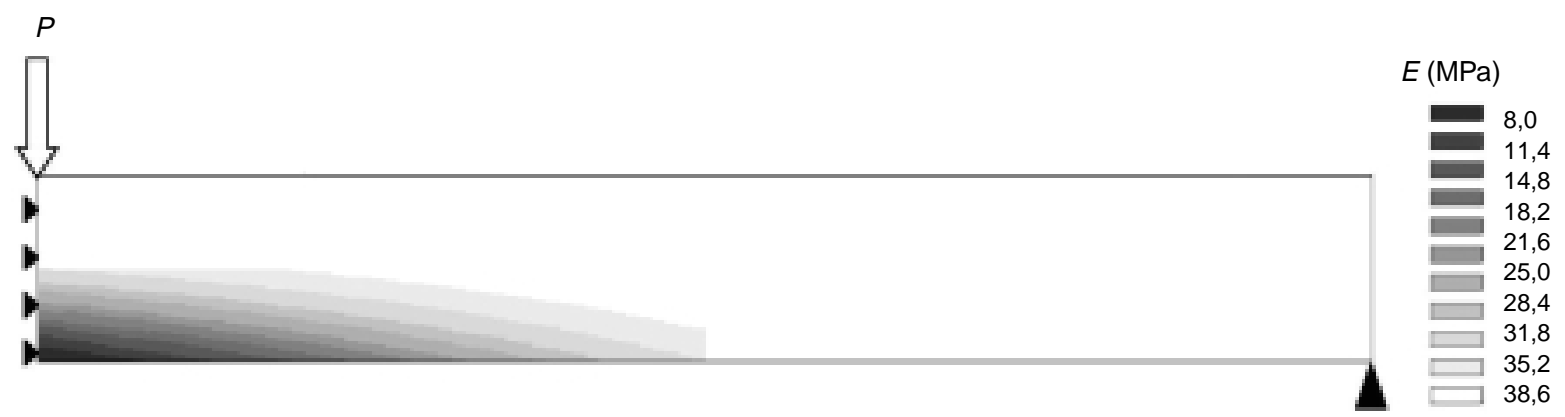

Figure $5 b$

Modèle de poutre endommagée.

Model for the damaged beam. 
des résistances à rupture des fibres (éq. (6)). Ce modèle n'est donc pas à proprement parler un modèle d'endommagement puisque les perturbations du champ de déformation liées au développement de l'endommagement ne sont pas prises en compte. Toutefois, ce calcul simplifié aboutit à une relation linéaire entre la raideur et le nombre de ruptures dans le VER (fig. 5a) avec des ordres de grandeur cohérents avec l'expérience. On écrira donc :

$$
\frac{R}{R_{0}}=1-\alpha N_{r}=\left(1-\alpha N_{t}\right)+\alpha P_{s} N_{t}
$$

$\alpha$ étant une constante empirique de l'ordre de $1,5 \cdot 10^{-3}$. En substituant la relation (6) dans l'équation (14), on obtient ainsi l'expression de la raideur relative sous sollicitation monotone :

$$
\frac{R}{R_{0}}(\varepsilon)=\left(1-\alpha N_{t}\right)+\alpha \exp \left(-\left(\frac{\varepsilon}{\varepsilon_{0}}\right)^{m}\right) N_{t}
$$

De même, l'équation (8) combinée à l'équation (14) conduit à l'expression suivante sous sollicitation périodique $(r \neq 1 ; \lambda<1)$ ou quasi statique $\left(r=1 ; \varepsilon_{\max }=\varepsilon_{\mathrm{app}} ; \lambda=1\right)$ :

$$
\begin{aligned}
\frac{R}{R_{0}}(t) & =\left(1-\alpha N_{t}\right) \\
& +\alpha \exp \left[-t^{m / n-2} \varepsilon_{\max }^{m n / n-2} \lambda^{m / n-2} k\right] N_{t} ;
\end{aligned}
$$

la valeur asymptotique de la raideur résiduelle, $R_{\infty}$, étant donnée par:

$$
\frac{R_{\infty}}{R_{0}}=\left(1-\alpha N_{t}\right)+\alpha \exp \left[-\left(\frac{\varepsilon_{\max } K_{I C}}{\varepsilon_{0} K_{I S}^{v}}\right)^{m}\right] N_{t}
$$

où $K_{I S}^{V}$ est le seuil de non-propagation des défauts dans le composite vieilli.

Dans une représentation de Weibull, nous avons constaté que les expressions (15) à (17) conduisent à des droites de pentes respectivement égales à $m$ et $m / n-2$ ainsi qu'à une limite asymptotique. Ces équations devraient donc pouvoir être simplifiées comme suit :

- Sollicitation monotone :

$$
\frac{R}{R_{0}}(\varepsilon) \approx \exp \left(-\left(\frac{\varepsilon_{\max }}{\varepsilon_{0}^{*}}\right)^{m}\right)
$$

où $\varepsilon_{0}{ }^{*}$ dépend du facteur d'échelle, $\varepsilon_{0}$, déterminé dans le VER et du paramètre $\alpha$.

- Sollicitation périodique ou quasi statique

$$
\frac{R}{R_{0}}(t) \approx \exp \left[-t^{m / n-2} \varepsilon_{\max }^{m n / n-2} \lambda^{m / n-2} k^{*}\right]
$$

puis

$$
\frac{R_{\infty}}{R_{0}} \approx \exp \left\lceil-\left(\frac{\varepsilon_{\max }}{\varepsilon^{*}}\right)^{m}\right\rceil
$$

où $k^{*}$ et $\varepsilon^{*}$ dépendent des facteurs déterminés à l'échelle du VER et du paramètre $\alpha$.

Les expressions ci-dessus présentent l'intérêt de reporter le paramètre $\alpha$ dans la valeur de la constante $k^{*}$, ici assimilée à un paramètre de calage du modèle et déterminée aisément par identification à partir de mesures macroscopiques de raideur. On obtient ainsi des équations cinétiques de la perte de raideur ne nécessitant plus l'évaluation préalable de la relation entre densité de ruptures et perte de raideur.

\section{ANALYSE PRÉDICTIVE DE LA PERTE DE RAIDEUR DE POUTRES COURBES}

Dans une dernière étape, le modèle de CSC a été appliqué à des éprouvettes courbes prélevées sur les structures composites flexibles à l'origine de l'étude. Celles-ci ont été caractérisées en flexion trois points sous des chargements de complexité croissante (monotone, relaxation, fatigue cyclique, fatigue à programme) permettant de valider le modèle de durabilité étape par étape. L'épaisseur de ces poutres ainsi que leurs conditions d'élaboration interdisant toute observation microscopique des mécanismes d'endommagement, l'analyse a donc été focalisée sur le suivi de raideur qui constitue un des paramètres pertinents dans une optique de dimensionnement. Comme pour le composite modèle, les hypothèses du modèle imposaient de restreindre son application aux étapes d'endommagement microscopique, c'est-àdire à des pertes de raideur n'excédant pas 5 à $10 \%$.

\subsection{Identification des paramètres du modèle}

La relation micro/macro établie au paragraphe précédent indique que la perte de raideur de la poutre doit permettre d'accéder aux informations relatives à la distribution des caractéristiques à rupture des fibres (à savoir, le module de Weibull $m$ ) et à la loi de propagation sous-critique (l'exposant $n$ ). Selon la relation (18), la représentation dans un plan de Weibull de la raideur d'une éprouvette sous chargement monotone doit ainsi conduire à la détermination du paramètre $m$. La figure 6, obtenue par flexion trois points d'une éprouvette non vieillie, permet de constater que la relation $\log \left(\ln \left(R_{0} / R\right)\right)=f(\log (\varepsilon))$ est effectivement une droite dont la pente donne une estimation cohérente du module de Weibull : $m=4,30 \pm 0,15$.

Une détermination fiable de l'exposant $n$ requiert l'établissement d'une courbe $v\left(K_{I}\right)$ dans les conditions de vieillissement considérées. De façon similaire à ce qui a été pratiqué à l'échelle du VER, nous avons procédé, par appariement des distributions de déformation à rupture et de temps à rupture associés, respectivement à un essai monotone sur composite non vieilli et un essai de relaxation sur composite préalablement conditionné en immersion. Afin d'éviter les problèmes liés à l'apparition d'un endommagement macroscopique 


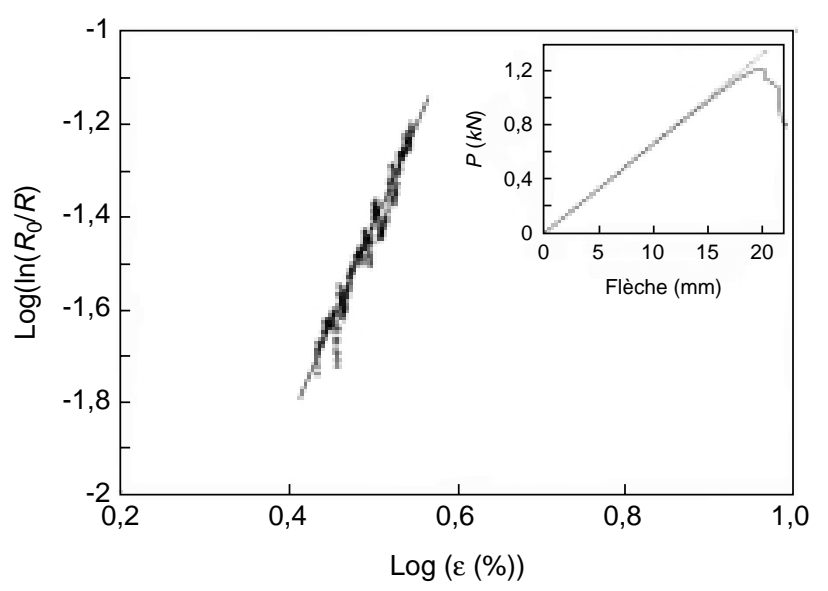

Figure 6

Représentation de Weibull de la perte de raideur d'une poutre composite non vieillie soumise à une sollicitation monotone. En insert : courbe force/déplacement.

Weibull plot of the stiffness loss of an unaged composite beam under monotonic loading. Insert box: load/displacement raw data.

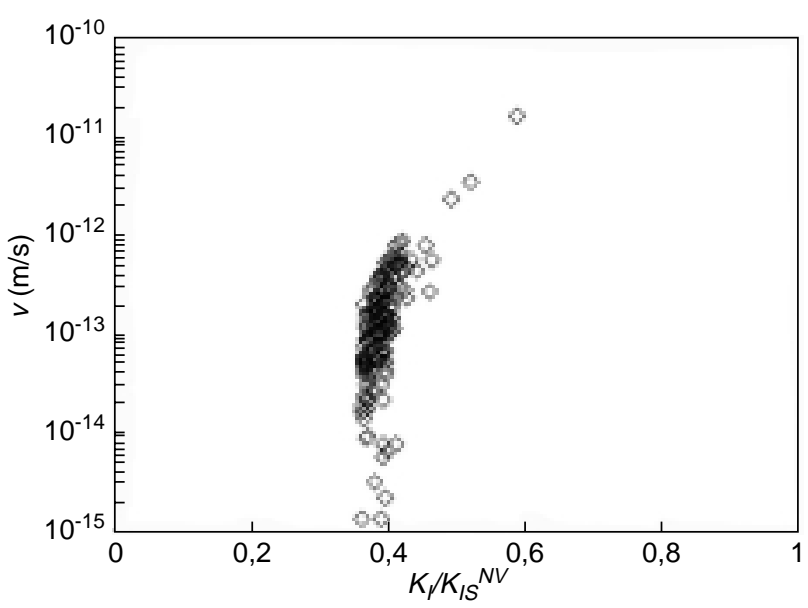

Figure 7

Courbe $v\left(K_{I}\right)$ des fibres de verre $\mathrm{E}_{\mathrm{CR}}{ }^{\circledR}$ obtenue par des mesures de raideur sur éprouvettes composites vieillies en immersion à $25^{\circ} \mathrm{C}$

$v\left(K_{I}\right)$ relationship for $E_{C R}{ }^{\circledR}$ glass fibres obtained from stiffness measurement using composites specimens aged at $25^{\circ} \mathrm{C}$ in water. local, la courbe $v\left(K_{I}\right)$ doit être tracée sous une déformation modérée $\left(\varepsilon_{\max }=1,2 \%\right)$. En dépit d'une dispersion relativement importante, la zone de seuil et le stade I apparaissent clairement (fig. 7). Le seuil $K_{I S} / K_{I S}{ }^{N V}$ (où $K_{I S}{ }^{N V}$ est le seuil de propagation du composite non vieilli) peut être estimé à 0,37 , et le coefficient de corrosion sous contrainte, $n$, est proche de 14 , soit une valeur proche de celle déterminée sur les éprouvettes planes de composite modèle.

\subsection{Essais de relaxation}

La figure 8 confronte, dans une représentation de Weibull et pour une sollicitation de relaxation, les cinétiques de perte de raideur expérimentales et théoriques. Ces dernières ont été établies à partir des données recueillies sur la courbe $v\left(K_{I}\right)$ et de l'essai à 1,2\% de déformation, non considéré dans cette figure. D'un point de vue quantitatif, on observe que la prévision est globalement proche de l'expérience. L'écart étant soit positif soit négatif, on peut penser qu'il est dû moins à une mauvaise estimation des paramètres du modèle, qu'à une incertitude sur la déformation imposée (ce que confirme le calcul de la barrette d'erreur avec une incertitude absolue sur la mesure de $\varepsilon_{\text {max }}$ de $0,05 \%$ ).

D'un point de vue qualitatif, on observe systématiquement dans la représentation de Weibull une zone approximativement linéaire de pente $0,33 \pm 0,05$. Pour les plus deux faibles sollicitations $(1,4$ et $1,6 \%)$, la cinétique de perte de raideur tend ensuite à ralentir puis redémarre dans un troisième temps. Compte tenu de la dégradation macroscopique de certaines des éprouvettes testées à 1,6\% de déformation, ceci

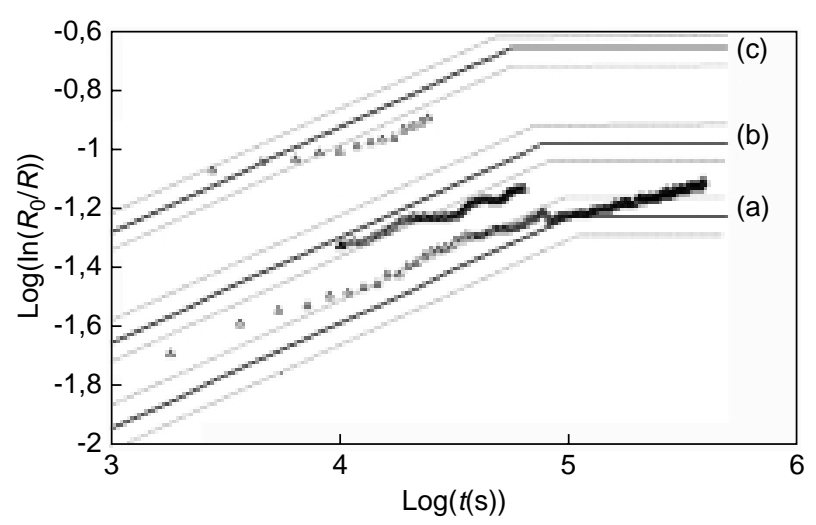

Figure 8

Cinétiques expérimentales $(\Delta)$ et théoriques (lignes continues) de perte de raideur sous sollicitation de relaxation. (a) $\varepsilon_{\max }=1,4 \%$; (b) $\varepsilon_{\text {max }}=1,6 \%$; (c) $\varepsilon_{\text {max }}=1,9 \%$.

(Les lignes pointillées correspondent à la prédiction pour un écart de $\pm 0,05 \%$ par rapport à la déformation nominale appliquée).

Experimental ( $\Delta)$ and theoretical (solid lines) stiffness loss curves under a relaxation condition.

(The dashed lines correspond to the model with a $\pm 0.05 \%$ deviation from the nominal applied strain).

peut être significatif du développement différé d'un endommagement local. Pour la sollicitation la plus forte $(1,9 \%)$, la rupture intervient avant même le ralentissement de la cinétique de perte de raideur. 
À ce stade, il paraît intéressant de déterminer si le modèle peut également prendre en compte d'éventuelles variations de température pouvant intervenir au cours de la vie en service de la structure. D'après la théorie générale des processus, la propagation sous-critique de fissures dans le verre est thermiquement activée. Expérimentalement, il a été largement vérifié que le coefficient $A$ de la loi de propagation sous-critique en stade I (éq. (1)) peut être écrit comme le produit d'un terme d'activation thermique et d'un terme dépendant des conditions hygrométriques $[8,14]$ :

$$
A=A_{\left[\mathrm{H}_{2} \mathrm{O}\right]} \exp \left(\frac{-E_{a}}{R T}\right)
$$

avec $T$ la température absolue, $R$ la constante des gaz parfaits et $E_{a}$ l'énergie d'activation.

Pour le verre de silice dans l'eau, l'énergie d'activation $E_{a}$ est de l'ordre de $130 \mathrm{~kJ} / \mathrm{mol}$ [14]. Pour le verre $E$ dans l'eau, elle varie de 60 à $100 \mathrm{~kJ} / \mathrm{mol}$ selon la teneur en ions alcalins [8]. Ceci conduit à une expression modifiée de la probabilité de survie au sein d'un écheveau sous sollicitation statique :

$$
P_{s}(t)=\exp \left[-t^{m / n-2} \varepsilon_{\text {app }}^{m n / n-2} \exp \left(\frac{-E_{a}}{R T}\right)^{m / n-2} k\right]
$$

Dans des conditions hygrométriques et physicochimiques données, l'ordonnée à l'origine, $F$, de la portion linéaire d'une représentation de Weibull de la raideur relative peut donc être décomposée de la façon suivante :

$$
\begin{aligned}
F & =m n / n-2 \log \left(\varepsilon_{\text {app }}\right) \\
& +0,434 m / n-2\left(\frac{-E a}{R T}\right)+\log \left(k^{*}\right)
\end{aligned}
$$

Nous avons procédé à des essais de relaxation sous 1,2\% de déformation imposée à différentes températures : 25, 40 et $60{ }^{\circ} \mathrm{C}$ (fig. 9a). La durée correspondant à la portion linéaire du tracé de Weibull est dans tous les cas inférieure à $8 \mathrm{~h}$, ce qui permet de négliger les effets de l'hydrolyse. Compte tenu de l'invariance de la prise d'eau à saturation dans la plage de température étudiée, nous avons considéré les conditions d'essai comme constantes à l'exception de la température. La figure $9 \mathrm{~b}$ présente l'évolution de $F$ en fonction de l'inverse de la température. Étant donné le nombre réduit de points dont nous disposons, la linéarité observée ne permet pas de conclure définitivement quant au caractère arrhénien de l'activation thermique. Cependant, la pente de cette droite fournit une estimation de l'énergie d'activation conforme aux références bibliographiques : $E_{a}=54 \mathrm{~kJ} / \mathrm{mol}$.

\subsection{Fatigue cyclique}

Selon les hypothèses de la CSC, la cinétique de rupture doit être indépendante de la fréquence et retardée par un débattement important (i.e., $r=\varepsilon_{\min } / \varepsilon_{\max }$, faible). Par ailleurs, les fibres ne connaissant jamais la rupture vérifient l'égalité quel que soit le type de sollicitation. D'après notre modèle, la probabilité de survie limite en fatigue doit donc être :

$$
P_{s}^{\infty}=\exp \left[-\left(\frac{\varepsilon_{\max } K_{I C}}{\varepsilon_{0} K_{I S}}\right)^{m}\right]
$$

Sous sollicitations cycliques, on peut toutefois s'attendre à rencontrer des phénomènes parasites venant interférer avec la CSC : la matrice et l'interface sont sujettes à une dégradation par fatigue qui conduit potentiellement à la perturbation des

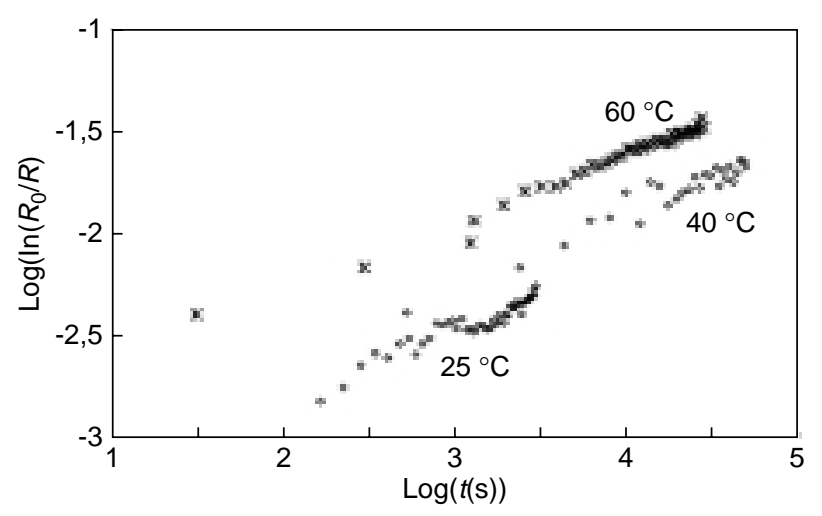

Figure 9a

Cinétiques de perte de raideur de poutres vieillies sollicitées en relaxation à différentes températures $\left(\varepsilon_{\max }=1,2 \%\right)$. Stiffness loss kinetics of aged composite beams under a relaxation condition at various temperatures $\left(\varepsilon_{\max }=1.2 \%\right)$.

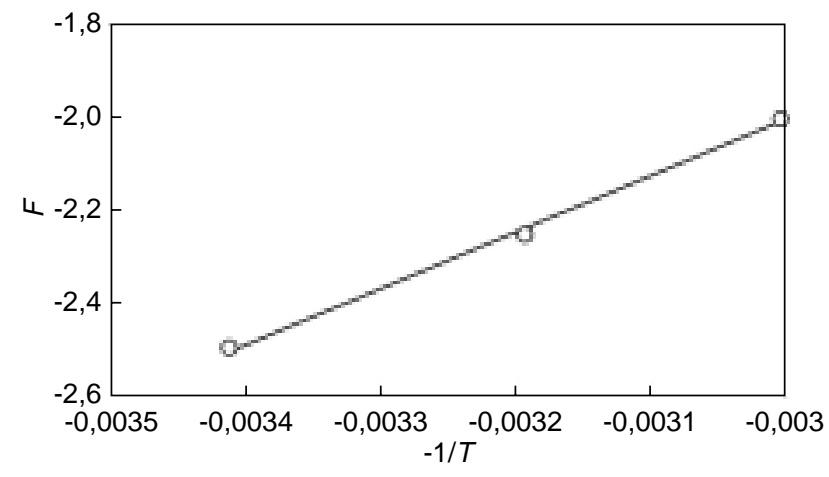

Figure 9b

Ordonnées à l'origine des droites $\log \left(\ln \left(R_{0} / R\right)=f(\log (t))\right.$ en fonction de l'inverse de la température.

Shift factor, $F$, of the stiffness loss kinetics as a function of temperature. 
champs de contraintes internes et de la cinétique de rupture des fibres. Ces mécanismes sont a priori accélérés par un rapport $r$ faible et sont largement dépendants du nombre de cycles. La cinétique de perte de raideur du composite est donc la résultante de deux mécanismes dépendants l'un, du temps (la CSC) et l'autre, du nombre de cycles. En conséquence, plus la fréquence $v$ sera élevée et le rapport $r$ faible, plus l'endommagement devra être logiquement dépendant du nombre de cycles. Inversement, plus la fréquence sera faible et le rapport $r$ élevé, plus l'endommagement sera théoriquement dépendant du temps et obéira aux hypothèses de la CSC. Chacun de ces points a été analysé dans le cas d'une sollicitation sinusoïdale en considérant les positions relatives d'essais statiques $(r=1)$ et cycliques $(r<1)$. Pour les différentes valeurs de $r$ et de $v$ considérées, la connaissance du spectre de chargement $\varepsilon(t)$ permet de calculer numériquement le facteur de retard $\lambda$ à partir de la seule estimation de $n$ (éq. (5)).

Le premier cas illustré est celui d'un cyclage à $r=0,7$ et $\varepsilon_{\max }=1,4 \%$. La figure 10 (a) présente, dans une échelle semi-logarithmique, la perte de raideur en fonction du nombre de cycles pour deux fréquences : 0,5 et $5 \mathrm{~Hz}$. La fréquence la plus faible semble la plus sévère et l'écart entre les deux courbes est largement supérieur aux incertitudes expérimentales (un quart de décade). La figure 10(b) présente les deux mêmes essais dans une échelle semi-logarithmique en fonction du temps. Il n'y a cette fois aucun écart appréciable, excepté au-delà de $5 \%$ de perte de raideur où la fréquence la plus forte semble la plus sévère. Par rapport à un essai de relaxation à $\varepsilon_{\text {app }}=1,4 \%$, on observe un retard significatif. Il est de l'ordre d'une décade tant que la probabilité de survie est supérieure à la valeur limite sous sollicitation statique $\left(P_{s}^{\infty}=0,94\right)$ et diminue ensuite car les essais cycliques ne présentent pas de limite de raideur apparente. Ce résultat permet de distinguer deux phases dans le mode d'endommagement : aux temps courts, la propagation sous-critique semble prédominante ; aux temps longs, la dégradation par fatigue devient au contraire le moteur de l'endommagement. Afin de valider le premier point, nous avons reporté dans la figure 11 la représentation de Weibull du début $\left(t<2 \cdot 10^{5} \mathrm{~s}\right)$ de ces mêmes essais de relaxation et de fatigue. Les portions de droite correspondantes sont parallèles et leur écart vertical $(F=-0,208)$ est conforme à la valeur déduite du modèle :

$$
m / n-2 \log \lambda=-0,209
$$

Le deuxième cas envisagé est celui d'un cyclage à $r=0,3$ et $\varepsilon_{\max }=1,4 \%$. La figure 12 présente, dans une échelle semilogarithmique, les cinétiques de perte de raideur en fonction du temps pour deux fréquences : 1 et $9 \mathrm{~Hz}$. Comme précédemment, la perte de raideur ne tend vers aucune valeur limite pour les temps considérés. Par rapport aux prédictions théoriques, l'essai à $9 \mathrm{~Hz}$ montre une légère avance alors que l'essai à $1 \mathrm{~Hz}$ présente un retard assez proche de celui prévu par le modèle de CSC jusqu'à une perte de raideur d'environ $5 \%$. On peut déduire des ces observations que la dégradation par fatigue est significative dès le début de l'essai pour la fréquence la plus forte $(9 \mathrm{~Hz})$ et qu'elle prend le relais de la CSC aux temps longs pour la plus faible $(1 \mathrm{~Hz})$.

Ces deux exemples montrent que le domaine de validité du modèle est a priori restreint à des fréquences faibles, des rapports $r$ élevés et des pertes de raideur n'excédant pas $5 \%$ environ. Sans qu'il nous ait été matériellement possible de borner de façon complète les limites du modèle en fonction des paramètres de sollicitation, les résultats que nous avons obtenus indiquent cependant que le spectre de houle auquel est soumis le matériau étudié coïncide a priori avec la plage de validité du modèle.

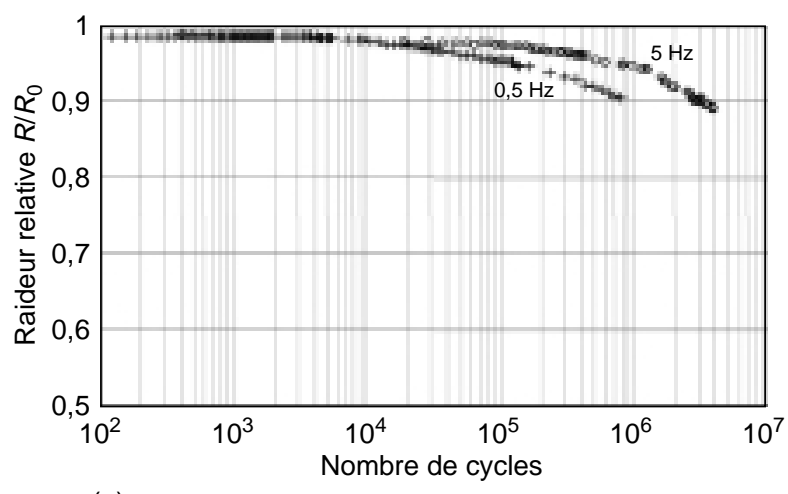

(a)

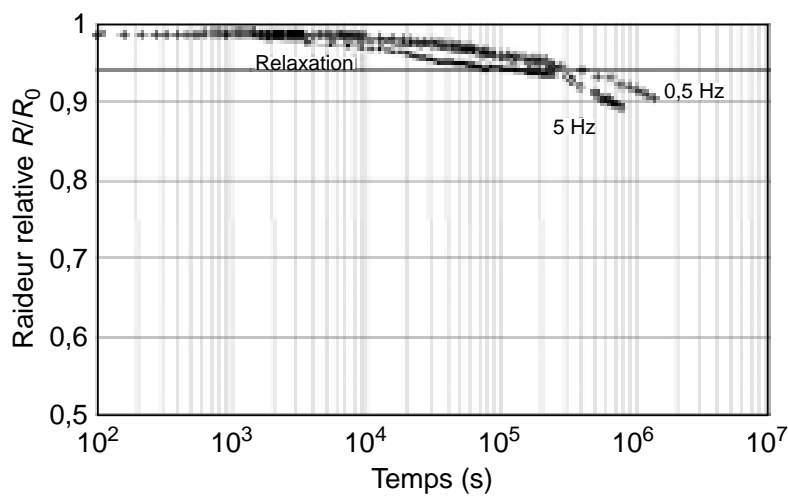

(b)

Figure 10

Cinétiques de perte de raideur à $0,5 \mathrm{~Hz}$ et $5 \mathrm{~Hz}$ en fonction du nombre de cycles (a) et du temps (b).

$\varepsilon_{\max }=1,7 \% ; r=0,7$.

Stiffness loss at $0,5 \mathrm{~Hz}$ and $5 \mathrm{~Hz}$ as a function of the number of fatigue cycles (a) and time (b). 


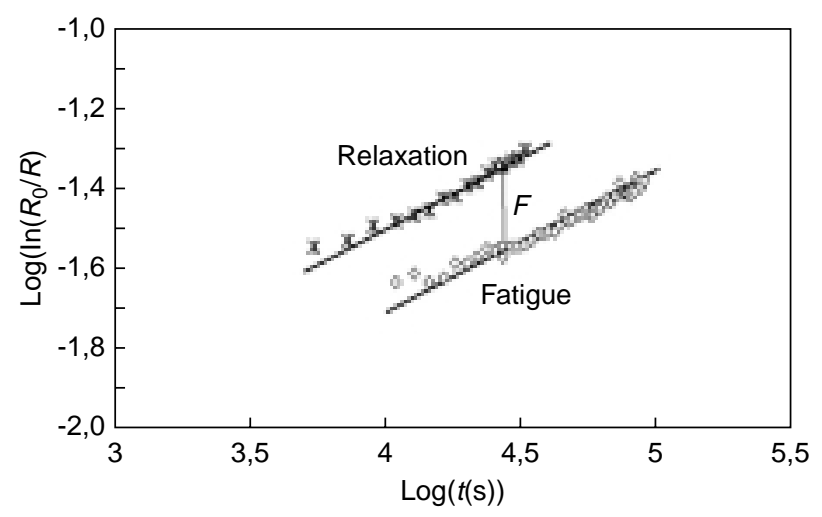

Figure 11

Comparaison entre les cinétiques de perte de raideur sous sollicitation de fatigue $\left(\varepsilon_{\max }=1,7 \% ; r=0.7 ; n=0,5\right.$ et $5 \mathrm{~Hz})$ et de relaxation $\left(\varepsilon_{\max }=1,7 \% ; \mathrm{r}=1\right)$.

Comparison between the stiffness loss kinetics under cyclic fatigue $\left(\varepsilon_{\max }=1.7 \% ; r=0.7 ; n=0.5\right.$ and $\left.5 \mathrm{~Hz}\right)$ and relaxation conditions $\left(\varepsilon_{\max }=1.7 \% ; r=1\right)$.

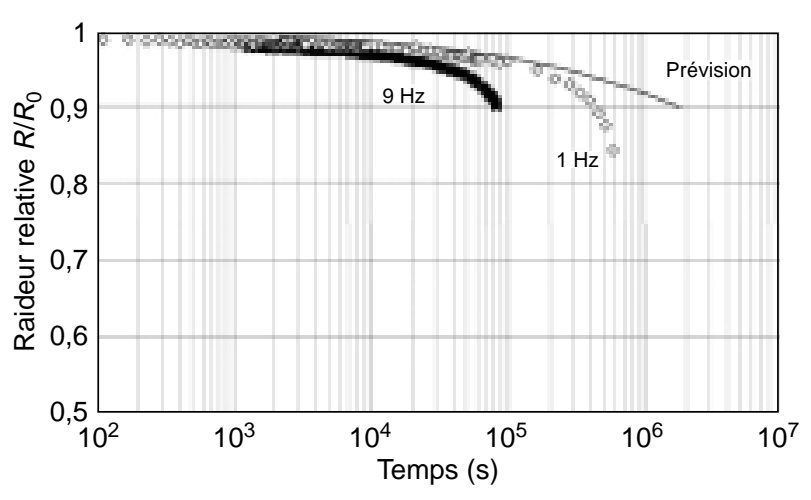

Figure 12

Cinétiques de perte de raideur à $1 \mathrm{~Hz}$ et $9 \mathrm{~Hz}$ pour un rapport de sollicitation faible $\left(r=0,3 ; \varepsilon_{\max }=1,4 \%\right)$.

Stiffness loss kinetics at $1 \mathrm{~Hz}$ and $9 \mathrm{~Hz}$ under a low $\mathrm{r}$ ratio condition $\left(r=0.3 ; \varepsilon_{\max }=1.4 \%\right)$.

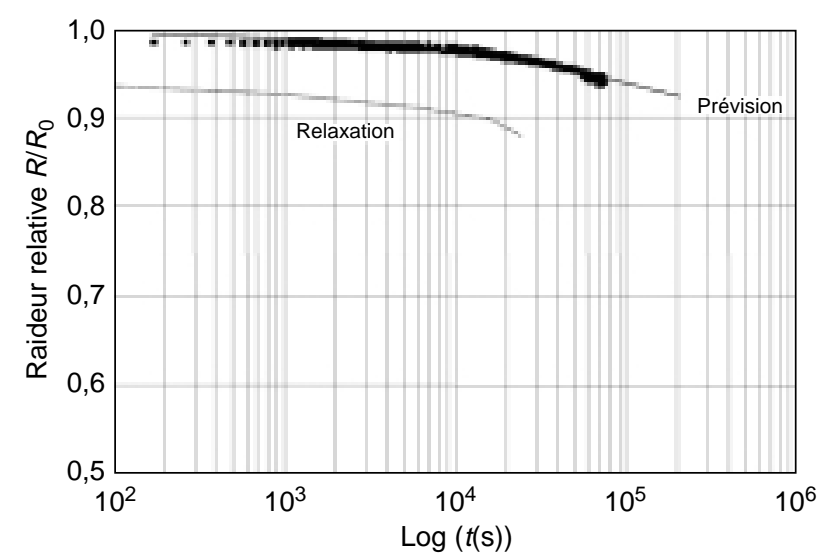

Figure 13

Courbes expérimentales et calculées de perte de raideur sous condition de fatigue à bloc.

(Les deux courbes expérimentales correspondent à deux essais distincts. En pointillés : rappel de l'essai de relaxation à $\varepsilon_{\max }=1,9 \%$ ).

Experimental and theoretical stiffness loss under stacking block sequence fatigue.

(The two experimental curves correspond to two separate tests. Dashed line: relaxation test for $\varepsilon_{\max }=1.9 \%$ ).

\subsection{Fatigue à blocs}

Dans la réalité, la majorité des applications présentent des structures travaillantes soumises à des chargements complexes intégrant des fluctuations du niveau de contrainte maximale, de la fréquence et du rapport de sollicitation. Théoriquement, le modèle développé doit permettre de prendre en compte de tels chargements au travers de la seule estimation du paramètre $\lambda$ (éq. (5)), dans la mesure, toutefois, où le chargement conserve son caractère périodique. Afin de valider le calcul de ce facteur de retard $\lambda$ pour un chargement complexe mais périodique, nous avons programmé l'asservissement du déplacement afin de répéter trois séquences sinusoïdales définies de sorte que les effets de la CSC soient prépondérants dans la perte de raideur. La figure 13 illustre les résultats obtenus pour la séquence suivante :

- séquence 1 (40 s) : $\varepsilon_{\max }=1,4 \%, r=0,7$ et $f=1 \mathrm{~Hz}$;

- séquence $2(40 \mathrm{~s}): \varepsilon_{\max }=1,2 \%, r=0,9$ et $f=3 \mathrm{~Hz}$;

- séquence 3 (20 s) : $\varepsilon_{\text {max }}=1,9 \%, r=0,8$ et $f=5 \mathrm{~Hz}$.

Pour les deux essais réalisés, la prévision est très proche de l'expérience, ce qui conforte la possibilité d'appliquer le modèle de CSC à la prévision de durée de vie de poutres soumises à des spectres de chargement plus complexes que la seule fatigue sous sollicitation sinusoïdale.

\section{CONCLUSION}

En nous fondant sur des concepts de corrosion sous contrainte, nous avons développé et validé un modèle de durabilité permettant de prédire la cinétique de perte de raideur de poutres composites à renfort verre soumises à des sollicitations de flexion en environnement humide. Le développement de ce modèle a été restreint aux stades initiaux de l'endommagement, pendant lesquels nous avons considéré que la perte de raideur provenait essentiellement de l'accumulation, à l'échelle microscopique, de ruptures différées de 
fibres sous l'action conjuguée de la contrainte et de l'humidité. Bien que limités, les niveaux de perte de raideur considérés (de 5 à $10 \%$ ) correspondent toutefois à la pratique du dimensionnement par fatigue de poutres travaillantes unidirectionnelles. Sur cette base, nous avons pu déterminer des expressions explicites de la perte de raideur de poutres composites à partir du calcul de la probabilité de survie des fibres. Ce dernier requiert principalement deux informations, à savoir, la distribution initiale des caractéristiques à rupture du renfort et la loi de propagation sous-critique des fissures dans les fibres selon les conditions d'environnement considérées au sein de la matrice vieillie.

Un des intérêts du modèle est de ne nécessiter que de l'identification d'un nombre réduit de paramètres accessibles à partir de caractérisations mécaniques simples, à savoir, des essais monotones à rupture et de relaxation. Au travers d'expérimentations en fatigue par bloc, nous avons démontré son aptitude à rendre compte des pertes de raideur sous des spectres de chargements relativement complexes, se rapprochant des conditions réelles de service de structure composite. Les résultats obtenus indiquent cependant que les mécanismes de corrosion sous contrainte ne dominent la réponse en fatigue qu'à l'intérieur d'une certaine plage de fréquence et de rapport de sollicitation, en dehors de laquelle des perturbations induites par le comportement cyclique de la matrice et des interfaces apparaissent. Les résultats recueillis démontrent toutefois que le domaine de validité du modèle de CSC recouvre largement le spectre de sollicitation de structures travaillantes offshore soumises à l'action de la houle.

Sous sollicitation de flexion, la localisation de l'endommagement dans les couches superficielles du composite nous a permis de définir de façon simple des protocoles de conditionnement permettant d'obtenir, à température modérée et en des temps courts (quelques dizaines de jours), un état de vieillissement homogène dans les zones du matériau affectées par la dégradation en fatigue. Dans le cas de sollicitations de traction, l'existence de transitoires de diffusion très longs dans l'épaisseur de la structure impliquerait de considérer un gradient de vieillissement induisant une distribution des paramètres de la loi de propagation sous-critique des défauts. Sous réserve d'identification de ces derniers, le modèle dans sa formulation actuelle doit théoriquement permettre de prendre en compte de telles hétérogénéités de vieillissement.

\section{RÉFÉRENCES}

1 Schutte, C. (1994) Environmental Durability of Glass-Fibre Composites. Mat. Sci. Eng. R, 13, 7, 265-323.

2 Talreja, R. (1987) Fatigue of Composite Materials. Basel, Technomic Publ. Co. Inc.

3 Vauthier, E., Abry, J.C., Bailliez, T. et Chateauminois, A. (1998) Interactions between Hygrothermal Ageing and Fatigue Damage in Unidirectionnal Glass/Epoxy Composites. Comp. Sci. Tech., 58, 687-692.
4 Chateauminois, A., Chabert, B., Soulier, J.P. et Vincent, L. (1993) Hygrothermal Ageing Effects on the Static Fatigue of Glass/Epoxy Composites. Composites, 24, 7, 547-555.

5 Jones, F.R., Rock, J.W. et Bailey, J.E. (1983) Stress Corrosion Cracking and its Implications for the Long Term Durability of E-Glass Fibre Composites. Composites. 14, 3 , 262-269.

6 Jones, F.R., Rock, J.W. et Bailey, J.E. (1983) The Environmental Stress Corrosion Cracking of Glass FibreReinforced Laminates and Single E-Glass Filaments. J. Mat. Sci., 18, 1059-1071.

7 Charles, R J. (1958) Static Fatigue of Glass-I. J. of Applied Physics, 29, 11, 1549.

8 Metcalfe, A.G. et Schmitz, G.K. (1972) Mechanism of Stress Corrosion in E-Glass Fibres. Glass Technology, 13, 1, 5-16

9 Aveston, J., Kelly, A. et Sillwood, J.M. (1980). Long Term Strength of Glass Feinforced Plastics in Wet Environments. Advances in Composite Materials. A.R. Bunsell, C. Bathias, A. Martrenchar, D. Menkes et G. Verchery, New York, Pergamon Press. 1, 556-568.

10 Cowking, A., Attou, A., Siddiqui, A.M. et Sweet, A.S. (1991) An Acoustic Emission Study of Failure by Stress Corrosion in Bundles of E-Glass Fibres. J. Mat. Sci., 26, 301-306.

11 Chateauminois, A. (2000) Interactions between Moisture and Flexural Fatigue Damage in Unidirectional Glass/Epoxy Composites. Recent Developments in Durability Analysis of Composite Systems, Cardon, Fukuda, Reifsnider and Verchery, Rotterdam, Balkema, 159-167.

12 Lü, B.T. (1997) Fatigue Strength Prediction of Soda-Lime Glass. Theoretical and Applied Fracture Mechanics, 27, 107114.

13 Wiederhorn, S.M. et Bolz, L.H. (1970) Stress Corrosion and Static Fatigue of Glass. J. of the American Ceramic Society, 53, 543-548.

14 Wiederhorn, S.M. (1978) Mechanisms of Subcritical Crack Growth in Glass. Fracture Mechanics of Ceramics, R.C. Bradt, Plenum Press. 4, 549-580.

15 Weibull, W. (1951) A Statistical Distribution Function of Wide Applicability. J. Appl. Mech., 18, 293-296.

16 Zinck, P., Pays, M.F., Rezakhanlou, R. et Gerard, J.F. (1999) Extrapolation Techniques at ShorGauge Lengths Based on the Weakest Link Concept for Fibres Exhibiting Multiple Failure Modes. Phil. Mag. A., 79, 9, 2103-2122.

17 Jakus, K., Coyne, D.C. et Ritter, J.E. (1978) Analysis of Fatigue Data for Lifetime Predictions for Ceramic Materials. J. Mat. Sci., 13, 2071-2080.

18 Muraoka, M., Ebata, K. et Abé, H. (1993) Effect of Humidity on Small-Crack Growth in Silica Optica Fibers, Journal of the American Ceramics Society, 76, 6, 1545-1550.

19 Fett, T. et Munz, D. (1993) Determination of $v-K_{I}$ Curves by a Modified Evaluation of Lifetime Measurements in Static Bending Tests. J. of the American Ceramic Society, 1985, 68, 8, C213-C215.

20 Pauchard, V., Brochado, S., Chateauminois, A., CampionBoulharts, H. et Grosjean, F. (2001) Measurements of SubCritical Crack Growth Rates in Glass Fibres by Means of Acoustic Emission. J. of Materials Science Letters, 20, 777779.

21 Pauchard, V. (2000) Méthode de caractérisation comportant des essais statiques et monotones sur mèches de fibres. Brevet français $n^{\circ}$ 00/01 230 .

22 Wiederhorn, S. M. (1978) Subcritical Crack Growth in Ceramics. Fracture Mechanics of Ceramics. R.C. Bradt, Plenum Press. 2. 
23 Pauchard, V. (2001) Étude des mécanismes de rupture dans les composites UD verre/époxy sous chargement de fatigue longitudinale en milieu humide : application d'un modèle de corrosion sous contrainte. Thèse de l'École centrale de Lyon.
24 Shen, C.H. et Springer, G.S. (1981) Moisture Absorption and Desorption of Composites Materials. Environmental Effects on Composites Materials, G.S. Springer, Lancaster, Technomic Pub., 15.

Manuscrit final reçu en octobre 2001 